Supporting Information for

\title{
Synthesis and Structural Investigation of
}

\section{Tungsten Imido Amidinate and Guanidinate \\ Complexes}

Corey B. Wilder, Khalil A. Abboud and Lisa McElwee-White* 


\section{Crystallographic data for $\mathrm{W}\left(\mathrm{N}^{\mathrm{i}} \mathrm{Pr}\right) \mathrm{Cl}_{3}\left[{ }^{\mathrm{i}} \mathrm{PrNC}\left(\mathrm{NMe}_{2}\right) \mathrm{N}^{\mathrm{i}} \mathrm{Pr}\right]$ (3)}

Table S1. Crystal data and structure refinement for 3.

Empirical formula

Formula weight

Temperature

Wavelength

Crystal system

Space group

Unit cell dimensions

Volume

Z

Density (calculated)

Absorption coefficient

$\mathrm{F}(000)$

Crystal size

Theta range for data collection

Index ranges

Reflections collected

Independent reflections

Completeness to theta $=24.60^{\circ}$

Absorption correction
C12 H27 Cl3 N4 W

517.58

173(2) K

$0.71073 \AA$

Triclinic

P-1

$a=8.4844(7) \AA$ $\alpha=93.063(2)^{\circ}$.

$\mathrm{b}=8.8717(8) \AA$ $\beta=101.094(2)^{\circ}$.

$\mathrm{c}=14.8556(13) \AA$ $\gamma=116.862(2)^{\circ}$.

$966.23(15) \AA^{3}$

2

$1.779 \mathrm{Mg} / \mathrm{m}^{3}$

$6.389 \mathrm{~mm}^{-1}$

504

$0.06 \times 0.05 \times 0.01 \mathrm{~mm}^{3}$

1.42 to $24.60^{\circ}$.

$-9 \leq \mathrm{h} \leq 9,-9 \leq \mathrm{k} \leq 9,-16 \leq \mathrm{l} \leq 16$

6201

$2732[\mathrm{R}(\mathrm{int})=0.0465]$

$83.6 \%$

Integration 
Max. and min. transmission

Refinement method

Data / restraints / parameters

Goodness-of-fit on $\mathrm{F}^{2}$

Final $\mathrm{R}$ indices $[\mathrm{I}>2 \operatorname{sigma}(\mathrm{I})]$

$\mathrm{R}$ indices (all data)

Largest diff. peak and hole

$\mathrm{R} 1=\sum\left(|| \mathrm{F}_{\mathrm{O}}|-| \mathrm{F}_{\mathrm{C}} \|\right) / \sum\left|\mathrm{F}_{\mathrm{O}}\right|$

$w R 2=\left[\sum\left[w\left(F_{\mathrm{O}}^{2}-\mathrm{F}^{2}\right)^{2}\right] / \sum\left[w\left(\mathrm{~F}_{\mathrm{O}}^{2}\right)^{2}\right]\right]^{1 / 2}$

$S=\left[\sum\left[w\left(F_{o}^{2}-F_{c}^{2}\right)^{2}\right] /(n-p)\right]^{1 / 2}$

$\mathrm{w}=1 /\left[\sigma^{2}\left(\mathrm{~F}_{\mathrm{O}}{ }^{2}\right)+(\mathrm{m} * \mathrm{p})^{2}+\mathrm{n} * \mathrm{p}\right], \mathrm{p}=\left[\max \left(\mathrm{F}_{\mathrm{O}}^{2}, 0\right)+2 * \mathrm{~F}_{\mathrm{c}}^{2}\right] / 3, \mathrm{~m} \& \mathrm{n}$ are constants.
Full-matrix least-squares on $\mathrm{F}^{2}$

$2732 / 1 / 189$

0.935

$\mathrm{R} 1=0.0264, \mathrm{wR} 2=0.0487[2299]$

$\mathrm{R} 1=0.0361, \mathrm{wR} 2=0.0502$

0.757 and -0.694 e. $\AA^{-3}$ 
Table S2. Atomic coordinates ( $\left.\mathrm{x} 10^{4}\right)$ and equivalent isotropic displacement parameters $\left(\AA^{2} \mathrm{x}\right.$ $10^{3}$ ) for 3 . $U(e q)$ is defined as one third of the trace of the orthogonalized $U^{i j}$ tensor.

\begin{tabular}{|c|c|c|c|c|}
\hline & $\mathrm{X}$ & $\mathrm{y}$ & $\mathrm{z}$ & $\mathrm{U}(\mathrm{eq})$ \\
\hline W & $7016(1)$ & $7032(1)$ & $7632(1)$ & $26(1)$ \\
\hline $\mathrm{Cl1}$ & $5687(2)$ & $7915(2)$ & $8678(1)$ & $38(1)$ \\
\hline $\mathrm{Cl} 2$ & $8116(2)$ & $6483(2)$ & $6359(1)$ & $40(1)$ \\
\hline $\mathrm{Cl} 3$ & $8410(2)$ & $9951(2)$ & $7413(1)$ & $41(1)$ \\
\hline N1 & 4397(6) & $6343(5)$ & $6592(3)$ & $24(1)$ \\
\hline N2 & $5064(6)$ & $4661(5)$ & $7418(3)$ & $27(1)$ \\
\hline N3 & $8643(6)$ & $6959(5)$ & $8474(3)$ & $34(1)$ \\
\hline N4 & 2017(6) & $3424(5)$ & $6419(3)$ & $28(1)$ \\
\hline $\mathrm{C} 1$ & $3727(7)$ & $4783(7)$ & $6756(4)$ & $24(1)$ \\
\hline $\mathrm{C} 2$ & $399(7)$ & $3668(7)$ & $6198(5)$ & $45(2)$ \\
\hline C3 & $1760(8)$ & $1769(6)$ & $6008(4)$ & $38(2)$ \\
\hline $\mathrm{C} 4$ & $4696(8)$ & $3194(7)$ & $7934(4)$ & $37(2)$ \\
\hline C5 & $6139(8)$ & $2624(7)$ & $7907(5)$ & $51(2)$ \\
\hline C6 & $4637(12)$ & $3662(9)$ & $8917(5)$ & $76(3)$ \\
\hline $\mathrm{C} 7$ & $3574(7)$ & $6973(6)$ & $5844(4)$ & $26(1)$ \\
\hline C8 & $4842(8)$ & $7656(7)$ & $5194(4)$ & $39(2)$ \\
\hline C9 & $3186(8)$ & $8344(7)$ & $6261(4)$ & $40(2)$ \\
\hline $\mathrm{C} 10$ & $10305(16)$ & $7240(20)$ & $9166(9)$ & $60(5)$ \\
\hline C11 & $11945(17)$ & $8636(18)$ & $9104(9)$ & $74(6)$ \\
\hline
\end{tabular}




$\begin{array}{lrrrr}\text { C12 } & 10170(20) & 7930(20) & 10126(12) & 77(5) \\ \text { C10' } & 9830(20) & 6760(20) & 9284(8) & 15(4) \\ \mathrm{C}^{\prime} 1^{\prime} & 11400(30) & 6870(30) & 8919(17) & 109(11) \\ \mathrm{C} 12^{\prime} & 9780(30) & 7210(30) & 10141(17) & 71(8)\end{array}$


Table S3. Bond lengths $[\AA ̊]$ and angles $\left[{ }^{\circ}\right]$ for 3 .

\begin{tabular}{|c|c|}
\hline W-N3 & $1.702(4)$ \\
\hline W-N2 & $1.961(4)$ \\
\hline W-N1 & $2.247(4)$ \\
\hline W-Cl1 & $2.3752(15)$ \\
\hline $\mathrm{W}-\mathrm{Cl} 2$ & $2.3819(16)$ \\
\hline $\mathrm{W}-\mathrm{Cl} 3$ & $2.3833(14)$ \\
\hline N1-C1 & $1.294(6)$ \\
\hline N1-C7 & $1.468(6)$ \\
\hline $\mathrm{N} 2-\mathrm{C} 1$ & $1.399(6)$ \\
\hline $\mathrm{N} 2-\mathrm{C} 4$ & $1.488(6)$ \\
\hline N3-C10 & $1.485(8)$ \\
\hline N3-C10' & $1.488(8)$ \\
\hline $\mathrm{N} 4-\mathrm{C} 1$ & $1.373(6)$ \\
\hline N4-C3 & $1.463(6)$ \\
\hline $\mathrm{N} 4-\mathrm{C} 2$ & $1.464(6)$ \\
\hline $\mathrm{C} 2-\mathrm{H} 2 \mathrm{~A}$ & 0.9800 \\
\hline $\mathrm{C} 2-\mathrm{H} 2 \mathrm{~B}$ & 0.9800 \\
\hline $\mathrm{C} 2-\mathrm{H} 2 \mathrm{C}$ & 0.9800 \\
\hline C3-H3A & 0.9800 \\
\hline C3-H3B & 0.9800 \\
\hline C3-H3C & 0.9800 \\
\hline
\end{tabular}




\begin{tabular}{|c|c|}
\hline C4-C6 & $1.513(9)$ \\
\hline $\mathrm{C} 4-\mathrm{C} 5$ & $1.527(8)$ \\
\hline C4-H4A & 1.0000 \\
\hline C5-H5A & 0.9800 \\
\hline C5-H5B & 0.9800 \\
\hline C5-H5C & 0.9800 \\
\hline C6-H6A & 0.9800 \\
\hline C6-H6B & 0.9800 \\
\hline C6-H6C & 0.9800 \\
\hline $\mathrm{C} 7-\mathrm{C} 8$ & $1.527(7)$ \\
\hline C7-C9 & $1.526(7)$ \\
\hline C7-H7A & 1.0000 \\
\hline C8-H8A & 0.9800 \\
\hline C8-H8B & 0.9800 \\
\hline $\mathrm{C} 8-\mathrm{H} 8 \mathrm{C}$ & 0.9800 \\
\hline C9-H9A & 0.9800 \\
\hline C9-H9B & 0.9800 \\
\hline C9-H9C & 0.9800 \\
\hline C10-C11 & $1.408(16)$ \\
\hline $\mathrm{C} 10-\mathrm{C} 12$ & $1.57(2)$ \\
\hline C10-H10A & 1.0000 \\
\hline C11-H11A & 0.9800 \\
\hline C11-H11B & 0.9800 \\
\hline
\end{tabular}




\begin{tabular}{|c|c|}
\hline C11-H11C & 0.9800 \\
\hline $\mathrm{C} 12-\mathrm{H} 12 \mathrm{~A}$ & 0.9800 \\
\hline C12-H12B & 0.9800 \\
\hline C12-H12C & 0.9800 \\
\hline C10'-C12' & $1.33(3)$ \\
\hline C10'-C11' & $1.50(3)$ \\
\hline C10'-H10B & 1.0000 \\
\hline C11'-H11D & 0.9800 \\
\hline C11'-H11E & 0.9800 \\
\hline C11'-H11F & 0.9800 \\
\hline C12'-H12D & 0.9800 \\
\hline C12'-H12E & 0.9800 \\
\hline C12'-H12F & 0.9800 \\
\hline N3-W-N2 & $101.44(19)$ \\
\hline N3-W-N1 & $163.23(18)$ \\
\hline N2-W-N1 & $61.88(16)$ \\
\hline N3-W-Cl1 & $95.27(17)$ \\
\hline N2-W-Cl1 & $91.76(13)$ \\
\hline N1-W-Cl1 & $84.12(12)$ \\
\hline N3-W-Cl2 & $95.84(17)$ \\
\hline $\mathrm{N} 2-\mathrm{W}-\mathrm{Cl} 2$ & $92.04(14)$ \\
\hline N1-W-Cl2 & $87.00(12)$ \\
\hline Cl1-W-Cl2 & $167.30(5)$ \\
\hline
\end{tabular}




\begin{tabular}{lc} 
N3-W-Cl3 & $102.74(15)$ \\
N2-W-Cl3 & $155.81(13)$ \\
N1-W-Cl3 & $93.92(11)$ \\
C11-W-Cl3 & $85.29(5)$ \\
C12-W-Cl3 & $86.29(5)$ \\
C1-N1-C7 & $126.1(4)$ \\
C1-N1-W & $90.3(3)$ \\
C7-N1-W & $142.6(3)$ \\
C1-N2-C4 & $123.8(4)$ \\
C1-N2-W & $100.0(3)$ \\
C4-N2-W & $134.1(3)$ \\
C10-N3-C10' & $19.6(8)$ \\
C10-N3-W & $168.4(8)$ \\
C10'-N3-W & $171.1(8)$ \\
C1-N4-C3 & $121.2(4)$ \\
C1-N4-C2 & $121.5(4)$ \\
C3-N4-C2 & $114.9(5)$ \\
N1-C1-N4 & $130.2(5)$ \\
N1-C1-N2 & $107.8(4)$ \\
N4-C1-N2 & $121.9(5)$ \\
N4-C2-H2A & 109.5 \\
H2-H2B & 109.5 \\
\hline
\end{tabular}




\begin{tabular}{|c|c|}
\hline $\mathrm{N} 4-\mathrm{C} 2-\mathrm{H} 2 \mathrm{C}$ & 109.5 \\
\hline $\mathrm{H} 2 \mathrm{~A}-\mathrm{C} 2-\mathrm{H} 2 \mathrm{C}$ & 109.5 \\
\hline $\mathrm{H} 2 \mathrm{~B}-\mathrm{C} 2-\mathrm{H} 2 \mathrm{C}$ & 109.5 \\
\hline N4-C3-H3A & 109.5 \\
\hline N4-C3-H3B & 109.5 \\
\hline $\mathrm{H} 3 \mathrm{~A}-\mathrm{C} 3-\mathrm{H} 3 \mathrm{~B}$ & 109.5 \\
\hline N4-C3-H3C & 109.5 \\
\hline $\mathrm{H} 3 \mathrm{~A}-\mathrm{C} 3-\mathrm{H} 3 \mathrm{C}$ & 109.5 \\
\hline H3B-C $3-\mathrm{H} 3 \mathrm{C}$ & 109.5 \\
\hline N2-C4-C6 & $110.9(5)$ \\
\hline $\mathrm{N} 2-\mathrm{C} 4-\mathrm{C} 5$ & $108.7(5)$ \\
\hline C6-C4-C5 & $112.3(5)$ \\
\hline N2-C4-H4A & 108.3 \\
\hline C6-C4-H4A & 108.3 \\
\hline C5-C4-H4A & 108.3 \\
\hline C4-C5-H5A & 109.5 \\
\hline C4-C5-H5B & 109.5 \\
\hline H5A-C5-H5B & 109.5 \\
\hline C4-C5-H5C & 109.5 \\
\hline $\mathrm{H} 5 \mathrm{~A}-\mathrm{C} 5-\mathrm{H} 5 \mathrm{C}$ & 109.5 \\
\hline H5B-C5-H5C & 109.5 \\
\hline C4-C6-H6A & 109.5 \\
\hline C4-C6-H6B & 109.5 \\
\hline
\end{tabular}




\begin{tabular}{|c|c|}
\hline H6A-C6-H6B & 109.5 \\
\hline C4-C6-H6C & 109.5 \\
\hline H6A-C6-H6C & 109.5 \\
\hline H6B-C6-H6C & 109.5 \\
\hline N1-C7-C8 & $109.4(4)$ \\
\hline N1-C7-C9 & $109.7(4)$ \\
\hline C8-C7-C9 & $111.8(4)$ \\
\hline N1-C7-H7A & 108.7 \\
\hline C8-C7-H7A & 108.7 \\
\hline C9-C7-H7A & 108.7 \\
\hline C7-C8-H8A & 109.5 \\
\hline C7-C8-H8B & 109.5 \\
\hline H8A-C8-H8B & 109.5 \\
\hline C7-C8-H8C & 109.5 \\
\hline $\mathrm{H} 8 \mathrm{~A}-\mathrm{C} 8-\mathrm{H} 8 \mathrm{C}$ & 109.5 \\
\hline H8B-C8-H8C & 109.5 \\
\hline C7-C9-H9A & 109.5 \\
\hline C7-C9-H9B & 109.5 \\
\hline H9A-C9-H9B & 109.5 \\
\hline C7-C9-H9C & 109.5 \\
\hline H9A-C9-H9C & 109.5 \\
\hline H9B-C9-H9C & 109.5 \\
\hline C11-C10-N3 & $116.0(12)$ \\
\hline
\end{tabular}




\begin{tabular}{|c|c|}
\hline C11-C10-C12 & $98.6(12)$ \\
\hline N3-C10-C12 & $106.6(11)$ \\
\hline C11-C10-H10A & 111.6 \\
\hline N3-C10-H10A & 111.6 \\
\hline C12-C10-H10A & 111.6 \\
\hline $\mathrm{C} 12^{\prime}-\mathrm{C} 10^{\prime}-\mathrm{N} 3$ & $119.9(15)$ \\
\hline C12'-C10'-C11' & $129.7(16)$ \\
\hline N3-C10'-C11' & $103.5(13)$ \\
\hline C12'-C10'-H10B & 98.6 \\
\hline N3-C10'-H10B & 98.6 \\
\hline C11'-C10'-H10B & 98.6 \\
\hline C10'-C11'-H11D & 109.5 \\
\hline C10'-C11'-H11E & 109.5 \\
\hline H11D-C11'-H11E & 109.5 \\
\hline C10'-C11'-H11F & 109.5 \\
\hline H11D-C11'-H11F & 109.5 \\
\hline H11E-C11'-H11F & 109.5 \\
\hline C10'-C12'-H12D & 109.5 \\
\hline C10'-C12'-H12E & 109.5 \\
\hline H12D-C12'-H12E & 109.5 \\
\hline C10'-C12'-H12F & 109.5 \\
\hline H12D-C12'-H12F & 109.5 \\
\hline H12E-C12'-H12F & 109.5 \\
\hline
\end{tabular}


Table S4. Anisotropic displacement parameters $\left(\AA^{2} \times 10^{3}\right)$ for 3 . The anisotropic displacement factor exponent takes the form: $-2 \pi^{2}\left[h^{2} a^{* 2} U^{11}+\ldots+2 h k a^{*} b^{*} U^{12}\right]$

\begin{tabular}{|c|c|c|c|c|c|c|}
\hline & $\mathrm{U}^{11}$ & $U^{22}$ & $\mathrm{U}^{33}$ & $U^{23}$ & $\mathrm{U}^{13}$ & $\mathrm{U}^{12}$ \\
\hline W & $24(1)$ & $26(1)$ & $27(1)$ & $5(1)$ & $3(1)$ & $12(1)$ \\
\hline Cl1 & $42(1)$ & $42(1)$ & $33(1)$ & $3(1)$ & 11(1) & $21(1)$ \\
\hline $\mathrm{Cl} 2$ & $37(1)$ & $47(1)$ & $41(1)$ & $5(1)$ & $14(1)$ & $24(1)$ \\
\hline $\mathrm{Cl} 3$ & $41(1)$ & $28(1)$ & $47(1)$ & $8(1)$ & $13(1)$ & $9(1)$ \\
\hline N1 & $25(3)$ & $20(3)$ & $26(3)$ & $9(2)$ & $2(2)$ & $12(2)$ \\
\hline N2 & $30(3)$ & $23(3)$ & $24(3)$ & $4(2)$ & $1(2)$ & $13(2)$ \\
\hline N3 & $30(3)$ & $34(3)$ & $34(3)$ & $3(2)$ & $-5(2)$ & $17(2)$ \\
\hline N4 & $28(3)$ & $26(3)$ & $28(3)$ & $6(2)$ & $3(2)$ & $13(2)$ \\
\hline $\mathrm{C} 1$ & $23(3)$ & $26(3)$ & $25(3)$ & $4(3)$ & $6(3)$ & $13(3)$ \\
\hline $\mathrm{C} 2$ & $23(3)$ & $43(4)$ & 71(5) & 24(4) & $9(3)$ & $16(3)$ \\
\hline $\mathrm{C} 3$ & $39(4)$ & $25(3)$ & $38(4)$ & 1(3) & $9(3)$ & $7(3)$ \\
\hline $\mathrm{C} 4$ & $44(4)$ & $31(4)$ & $30(4)$ & $10(3)$ & $-3(3)$ & $18(3)$ \\
\hline $\mathrm{C} 5$ & $60(5)$ & $39(4)$ & $59(5)$ & $13(4)$ & $-1(4)$ & $32(4)$ \\
\hline C6 & $134(8)$ & $65(5)$ & $49(5)$ & $37(4)$ & $44(5)$ & $53(5)$ \\
\hline $\mathrm{C} 7$ & 24(3) & 24(3) & $26(4)$ & 11(3) & 1(3) & $9(3)$ \\
\hline C8 & $51(4)$ & $40(4)$ & $28(4)$ & $14(3)$ & $5(3)$ & $23(3)$ \\
\hline C9 & $45(4)$ & $34(4)$ & $49(4)$ & $13(3)$ & $13(3)$ & $25(3)$ \\
\hline
\end{tabular}


Table S5. Hydrogen coordinates $\left(\times 10^{4}\right)$ and isotropic displacement parameters $\left(\AA^{2} \times 10^{3}\right)$ for 3 .

\begin{tabular}{|c|c|c|c|c|}
\hline & $\mathrm{x}$ & $\mathrm{y}$ & $\mathrm{z}$ & $\mathrm{U}(\mathrm{eq})$ \\
\hline $\mathrm{H} 2 \mathrm{~A}$ & 658 & 4759 & 6545 & 68 \\
\hline $\mathrm{H} 2 \mathrm{~B}$ & -614 & 2729 & 6371 & 68 \\
\hline $\mathrm{H} 2 \mathrm{C}$ & 70 & 3681 & 5530 & 68 \\
\hline $\mathrm{H} 3 \mathrm{~A}$ & 1313 & 1600 & 5332 & 56 \\
\hline H3B & 871 & 852 & 6262 & 56 \\
\hline $\mathrm{H} 3 \mathrm{C}$ & 2925 & 1745 & 6155 & 56 \\
\hline $\mathrm{H} 4 \mathrm{~A}$ & 3480 & 2225 & 7609 & 44 \\
\hline H5A & 6170 & 2388 & 7261 & 77 \\
\hline H5B & 5839 & 1584 & 8189 & 77 \\
\hline $\mathrm{H} 5 \mathrm{C}$ & 7334 & 3534 & 8255 & 77 \\
\hline H6A & 5799 & 4649 & 9238 & 114 \\
\hline H6B & 4428 & 2688 & 9249 & 114 \\
\hline $\mathrm{H} 6 \mathrm{C}$ & 3647 & 3951 & 8902 & 114 \\
\hline H7A & 2395 & 5997 & 5482 & 31 \\
\hline H8A & 6016 & 8593 & 5545 & 59 \\
\hline H8B & 4294 & 8079 & 4695 & 59 \\
\hline $\mathrm{H} 8 \mathrm{C}$ & 5029 & 6734 & 4925 & 59 \\
\hline H9A & 2272 & 7831 & 6621 & 59 \\
\hline
\end{tabular}




\begin{tabular}{|c|c|c|c|c|}
\hline H9B & 2727 & 8825 & 5761 & 59 \\
\hline H9C & 4312 & 9254 & 6670 & 59 \\
\hline H10A & 10415 & 6167 & 9186 & 71 \\
\hline H11A & 12056 & 8585 & 8460 & 110 \\
\hline H11B & 12975 & 8584 & 9507 & 110 \\
\hline $\mathrm{H} 11 \mathrm{C}$ & 11948 & 9708 & 9302 & 110 \\
\hline $\mathrm{H} 12 \mathrm{~A}$ & 10928 & 7699 & 10628 & 116 \\
\hline H12B & 8902 & 7351 & 10172 & 116 \\
\hline $\mathrm{H} 12 \mathrm{C}$ & 10600 & 9164 & 10179 & 116 \\
\hline H10B & 9144 & 5488 & 9242 & 18 \\
\hline H11D & 11844 & 7858 & 8595 & 164 \\
\hline H11E & 11005 & 5821 & 8485 & 164 \\
\hline $\mathrm{H} 11 \mathrm{~F}$ & 12387 & 6996 & 9437 & 164 \\
\hline H12D & 9524 & 8176 & 10158 & 107 \\
\hline $\mathrm{H} 12 \mathrm{E}$ & 10961 & 7534 & 10568 & 107 \\
\hline $\mathrm{H} 12 \mathrm{~F}$ & 8824 & 6236 & 10330 & 107 \\
\hline
\end{tabular}




\section{Crystallographic Data for $\mathrm{W}\left(\mathrm{N}^{\mathrm{i}} \mathrm{Pr}\right) \mathrm{Cl}_{3}\left[{ }^{\mathrm{i}} \operatorname{PrNC}(\mathrm{Me}) \mathrm{N}^{\mathrm{i}} \mathrm{Pr}\right](9)$}

Table S6. Crystal data and structure refinement for $\mathbf{9}$.

Empirical formula

Formula weight

Temperature

Wavelength

Crystal system

Space group

Unit cell dimensions

Volume

Z

Density (calculated)

Absorption coefficient

$\mathrm{F}(000)$

Crystal size

Theta range for data collection

Index ranges

Reflections collected

Independent reflections

Completeness to theta $=27.28^{\circ}$

Absorption correction

Max. and min. transmission
C11 H24 Cl3 N3 W

488.53

173(2) K

$0.71073 \AA$

Monoclinic

$\mathrm{P} 2(1) / \mathrm{c}$

$$
\begin{array}{ll}
\mathrm{a}=16.6234(11) \AA & \alpha=90^{\circ} . \\
\mathrm{b}=13.9173(9) \AA & \beta=114.095(2)^{\circ} . \\
\mathrm{c}=16.9854(11) \AA & \gamma=90^{\circ} .
\end{array}
$$

$3587.2(4) \AA^{3}$

8

$1.809 \mathrm{Mg} / \mathrm{m}^{3}$

$6.876 \mathrm{~mm}^{-1}$

1888

$0.22 \times 0.16 \times 0.06 \mathrm{~mm}^{3}$

1.97 to $27.28^{\circ}$.

$-20 \leq \mathrm{h} \leq 17,-17 \leq \mathrm{k} \leq 17,-21 \leq 1 \leq 20$

20476

$7210[\mathrm{R}(\mathrm{int})=0.0622]$

$89.6 \%$

Integration

0.6719 and 0.2461 
Refinement method

Data / restraints / parameters

Goodness-of-fit on $\mathrm{F}^{2}$

Final R indices $[\mathrm{I}>2 \operatorname{sigma}(\mathrm{I})]$

$\mathrm{R}$ indices (all data)

Largest diff. peak and hole

$\mathrm{R} 1=\sum\left(\left\|\mathrm{F}_{\mathrm{O}}|-| \mathrm{F}_{\mathrm{C}}\right\|\right) / \sum\left|\mathrm{F}_{\mathrm{O}}\right|$

$\mathrm{wR} 2=\left[\sum\left[\mathrm{w}\left(\mathrm{F}_{\mathrm{O}}{ }^{2}-\mathrm{F}_{\mathrm{c}}{ }^{2}\right)^{2}\right] / \sum\left[\mathrm{w}\left(\mathrm{F}_{\mathrm{O}}{ }^{2}\right)^{2}\right]\right]^{1 / 2}$

$S=\left[\sum\left[w\left(F_{O}^{2}-F_{c}^{2}\right)^{2}\right] /(n-p)\right]^{1 / 2}$

$\mathrm{w}=1 /\left[\sigma^{2}\left(\mathrm{~F}_{\mathrm{O}}^{2}\right)+\left(\mathrm{m}^{*} \mathrm{p}\right)^{2}+\mathrm{n} * \mathrm{p}\right], \mathrm{p}=\left[\max \left(\mathrm{F}_{\mathrm{O}}^{2}, 0\right)+2 * \mathrm{~F}^{2}\right] / 3, \mathrm{~m} \& \mathrm{n}$ are constants.
Full-matrix least-squares on $\mathrm{F}^{2}$

$7210 / 0 / 362$

1.041

$\mathrm{R} 1=0.0256, \mathrm{wR} 2=0.0632[6207]$

$\mathrm{R} 1=0.0325, \mathrm{wR} 2=0.0660$

0.780 and -1.068 e. $\AA^{-3}$ 
Table S7. Atomic coordinates ( $\left.\mathrm{x} 10^{4}\right)$ and equivalent isotropic displacement parameters $\left(\AA^{2} \mathrm{x}\right.$ $10^{3}$ ) for 9 . $\mathrm{U}(\mathrm{eq})$ is defined as one third of the trace of the orthogonalized $\mathrm{U}^{\mathrm{ij}}$ tensor.

\begin{tabular}{|c|c|c|c|c|}
\hline & $\mathrm{X}$ & $\mathrm{y}$ & $\mathrm{z}$ & $\mathrm{U}(\mathrm{eq})$ \\
\hline W1 & $4546(1)$ & $8040(1)$ & $3696(1)$ & $30(1)$ \\
\hline W2 & $9293(1)$ & $7530(1)$ & $8728(1)$ & $36(1)$ \\
\hline $\mathrm{Cl1}$ & $5683(1)$ & $9150(1)$ & $3840(1)$ & $39(1)$ \\
\hline $\mathrm{Cl} 2$ & $3695(1)$ & $6717(1)$ & 3809(1) & $57(1)$ \\
\hline $\mathrm{Cl} 3$ & 4998(1) & $8212(1)$ & $5204(1)$ & $54(1)$ \\
\hline $\mathrm{Cl} 4$ & $8250(1)$ & 8699(1) & $8738(1)$ & $58(1)$ \\
\hline $\mathrm{Cl} 5$ & 10046(1) & $6183(1)$ & $8488(1)$ & $59(1)$ \\
\hline $\mathrm{Cl} 6$ & $8738(1)$ & $7810(1)$ & $7222(1)$ & $51(1)$ \\
\hline N1 & $3671(2)$ & $8821(2)$ & $3283(2)$ & $35(1)$ \\
\hline N2 & $4600(2)$ & $7474(2)$ & $2658(2)$ & $33(1)$ \\
\hline N3 & $5589(2)$ & $6895(2)$ & $3851(2)$ & $30(1)$ \\
\hline N4 & $10108(10)$ & $8418(9)$ & $9070(11)$ & $40(3)$ \\
\hline N4' & $10279(10)$ & $8113(9)$ & $9169(10)$ & $32(3)$ \\
\hline N5 & $8222(2)$ & $6435(2)$ & $8602(2)$ & $37(1)$ \\
\hline N6 & $9294(2)$ & $6935(2)$ & $9776(2)$ & $37(1)$ \\
\hline $\mathrm{C} 1$ & $2926(3)$ & $9465(3)$ & $3019(3)$ & $42(1)$ \\
\hline $\mathrm{C} 2$ & $2075(3)$ & $8913(4)$ & $2673(5)$ & $90(2)$ \\
\hline C3 & $3016(4)$ & $10109(4)$ & $3758(4)$ & $84(2)$ \\
\hline $\mathrm{C} 4$ & $4102(3)$ & $7534(3)$ & $1715(3)$ & $44(1)$ \\
\hline
\end{tabular}




\begin{tabular}{|c|c|c|c|c|}
\hline $\mathrm{C} 5$ & $3887(3)$ & $8566(3)$ & $1428(3)$ & $54(1)$ \\
\hline C6 & $3296(4)$ & $6905(4)$ & $1437(3)$ & $77(2)$ \\
\hline C7 & $5293(3)$ & $6812(3)$ & $3030(2)$ & $33(1)$ \\
\hline $\mathrm{C} 8$ & $5590(3)$ & $6150(3)$ & $2514(3)$ & $43(1)$ \\
\hline C9 & $6324(3)$ & $6335(3)$ & $4482(3)$ & $39(1)$ \\
\hline $\mathrm{C} 10$ & $5947(3)$ & $5684(3)$ & $4968(3)$ & $53(1)$ \\
\hline $\mathrm{C} 11$ & $7013(3)$ & $7011(3)$ & $5074(3)$ & $49(1)$ \\
\hline $\mathrm{C} 12$ & $10777(7)$ & $9142(12)$ & $9296(7)$ & $51(3)$ \\
\hline $\mathrm{C} 12^{\prime}$ & $11125(11)$ & $8634(11)$ & $9430(10)$ & $58(3)$ \\
\hline C13 & $10695(9)$ & $9594(9)$ & $8470(8)$ & $78(4)$ \\
\hline C13' & 10937(19) & $9638(14)$ & $9360(30)$ & $220(20)$ \\
\hline $\mathrm{C} 14$ & $11667(8)$ & $8806(14)$ & $9841(15)$ & $134(11)$ \\
\hline C14' & $11620(10)$ & $8303(12)$ & $8988(13)$ & $107(7)$ \\
\hline $\mathrm{C} 15$ & $7446(3)$ & $5936(3)$ & $7972(3)$ & $51(1)$ \\
\hline $\mathrm{C} 16$ & $7748(4)$ & $5346(3)$ & $7384(3)$ & $68(2)$ \\
\hline $\mathrm{C} 17$ & $6752(3)$ & $6659(4)$ & $7482(3)$ & $60(1)$ \\
\hline $\mathrm{C} 18$ & $8558(2)$ & $6323(3)$ & $9422(2)$ & $33(1)$ \\
\hline C19 & $8277(3)$ & $5681(3)$ & $9965(3)$ & $46(1)$ \\
\hline $\mathrm{C} 20$ & $9841(3)$ & $6995(3)$ & $10715(3)$ & $48(1)$ \\
\hline $\mathrm{C} 21$ & $9917(4)$ & $8036(4)$ & $11006(3)$ & $62(1)$ \\
\hline $\mathrm{C} 22$ & $10729(4)$ & $6537(4)$ & 10913(3) & $74(2)$ \\
\hline
\end{tabular}


Table S8. Bond lengths $[\AA]$ and angles $\left[^{\circ}\right]$ for 9 .

\begin{tabular}{ll} 
W1-N1 & $1.720(3)$ \\
W1-N2 & $1.966(3)$ \\
W1-N3 & $2.290(3)$ \\
W1-Cl3 & $2.3679(10)$ \\
W1-Cl1 & $2.3750(9)$ \\
W1-Cl2 & $2.3754(11)$ \\
W1-C7 & $2.625(4)$ \\
W2-N4' & $1.705(16)$ \\
W2-N4 & $1.748(16)$ \\
W2-N6 & $1.964(3)$ \\
W2-N5 & $2.286(3)$ \\
W2-Cl6 & $2.3699(10)$ \\
W2-Cl5 & $2.3802(12)$ \\
W2-Cl4 & $2.3818(12)$ \\
W2-C18 & $2.624(4)$ \\
N1-C1 & $1.443(5)$ \\
N2-C7 & $1.407(5)$ \\
N2-C4 & $1.474(5)$ \\
N3-C7 & $1.281(5)$ \\
N3-C9 & $1.476(5)$ \\
N4-C12 & $1.43(2)$ \\
& \\
\hline
\end{tabular}




\begin{tabular}{|c|c|}
\hline $\mathrm{N} 4^{\prime}-\mathrm{C} 12^{\prime}$ & $1.48(2)$ \\
\hline N5-C18 & $1.282(5)$ \\
\hline N5-C15 & $1.471(5)$ \\
\hline N6-C18 & $1.408(5)$ \\
\hline N6-C20 & $1.480(5)$ \\
\hline $\mathrm{C} 1-\mathrm{C} 3$ & $1.500(6)$ \\
\hline $\mathrm{C} 1-\mathrm{C} 2$ & $1.501(6)$ \\
\hline C1-H1A & 1.0000 \\
\hline $\mathrm{C} 2-\mathrm{H} 2 \mathrm{~A}$ & 0.9800 \\
\hline $\mathrm{C} 2-\mathrm{H} 2 \mathrm{~B}$ & 0.9800 \\
\hline $\mathrm{C} 2-\mathrm{H} 2 \mathrm{C}$ & 0.9800 \\
\hline $\mathrm{C} 3-\mathrm{H} 3 \mathrm{~A}$ & 0.9800 \\
\hline $\mathrm{C} 3-\mathrm{H} 3 \mathrm{~B}$ & 0.9800 \\
\hline C3-H3C & 0.9800 \\
\hline C4-C6 & $1.506(7)$ \\
\hline $\mathrm{C} 4-\mathrm{C} 5$ & $1.512(6)$ \\
\hline C4-H4A & 1.0000 \\
\hline $\mathrm{C} 5-\mathrm{H} 5 \mathrm{~A}$ & 0.9800 \\
\hline C5-H5B & 0.9800 \\
\hline $\mathrm{C} 5-\mathrm{H} 5 \mathrm{C}$ & 0.9800 \\
\hline C6-H6A & 0.9800 \\
\hline C6-H6B & 0.9800 \\
\hline C6-H6C & 0.9800 \\
\hline
\end{tabular}




\begin{tabular}{|c|c|}
\hline C7-C8 & $1.488(5)$ \\
\hline C8-H8A & 0.9800 \\
\hline C8-H8B & 0.9800 \\
\hline C8-H8C & 0.9800 \\
\hline C9-C11 & $1.506(6)$ \\
\hline C9-C10 & $1.523(6)$ \\
\hline C9-H9A & 1.0000 \\
\hline $\mathrm{C} 10-\mathrm{H} 10 \mathrm{~A}$ & 0.9800 \\
\hline C10-H10B & 0.9800 \\
\hline $\mathrm{C} 10-\mathrm{H} 10 \mathrm{C}$ & 0.9800 \\
\hline C11-H11A & 0.9800 \\
\hline C11-H11B & 0.9800 \\
\hline $\mathrm{C} 11-\mathrm{H} 11 \mathrm{C}$ & 0.9800 \\
\hline C12-C14 & $1.465(16)$ \\
\hline C12-C13 & $1.491(15)$ \\
\hline $\mathrm{C} 12-\mathrm{H} 12 \mathrm{~A}$ & 1.0000 \\
\hline C12'-C14' & $1.399(18)$ \\
\hline C12'-C13' & $1.43(2)$ \\
\hline C12'-H12B & 1.0000 \\
\hline C13-H13A & 0.9800 \\
\hline C13-H13B & 0.9800 \\
\hline C13-H13C & 0.9800 \\
\hline C13'-H13D & 0.9800 \\
\hline
\end{tabular}




\begin{tabular}{|c|c|}
\hline C13'-H13E & 0.9800 \\
\hline C13'-H13F & 0.9800 \\
\hline C14-H14A & 0.9800 \\
\hline C14-H14B & 0.9800 \\
\hline C14-H14C & 0.9800 \\
\hline C14'-H14D & 0.9800 \\
\hline C14'-H14E & 0.9800 \\
\hline C14'-H14F & 0.9800 \\
\hline C15-C17 & $1.501(7)$ \\
\hline $\mathrm{C} 15-\mathrm{C} 16$ & $1.528(7)$ \\
\hline C15-H15A & 1.0000 \\
\hline C16-H16A & 0.9800 \\
\hline C16-H16B & 0.9800 \\
\hline C16-H16C & 0.9800 \\
\hline C17-H17A & 0.9800 \\
\hline C17-H17B & 0.9800 \\
\hline $\mathrm{C} 17-\mathrm{H} 17 \mathrm{C}$ & 0.9800 \\
\hline C18-C19 & $1.489(5)$ \\
\hline C19-H19A & 0.9800 \\
\hline C19-H19B & 0.9800 \\
\hline C19-H19C & 0.9800 \\
\hline C20-C22 & $1.515(7)$ \\
\hline C20-C21 & $1.519(6)$ \\
\hline
\end{tabular}




\begin{tabular}{|c|c|}
\hline C20-H20A & 1.0000 \\
\hline C21-H21A & 0.9800 \\
\hline $\mathrm{C} 21-\mathrm{H} 21 \mathrm{~B}$ & 0.9800 \\
\hline $\mathrm{C} 21-\mathrm{H} 21 \mathrm{C}$ & 0.9800 \\
\hline C22-H22A & 0.9800 \\
\hline $\mathrm{C} 22-\mathrm{H} 22 \mathrm{~B}$ & 0.9800 \\
\hline $\mathrm{C} 22-\mathrm{H} 22 \mathrm{C}$ & 0.9800 \\
\hline N1-W1-N2 & $103.16(14)$ \\
\hline N1-W1-N3 & $164.06(13)$ \\
\hline N2-W1-N3 & $60.95(12)$ \\
\hline N1-W1-Cl3 & $102.69(11)$ \\
\hline N2-W1-Cl3 & $154.12(9)$ \\
\hline N3-W1-Cl3 & $93.17(8)$ \\
\hline N1-W1-C11 & $97.78(10)$ \\
\hline N2-W1-C11 & $91.54(10)$ \\
\hline N3-W1-C11 & $84.66(8)$ \\
\hline Cl3-W1-Cl1 & $86.29(4)$ \\
\hline N1-W1-Cl2 & $95.22(11)$ \\
\hline N2-W1-C12 & $90.50(10)$ \\
\hline N3-W1-Cl2 & $84.25(8)$ \\
\hline Cl3-W1-C12 & $85.84(4)$ \\
\hline Cl1-W1-C12 & $166.01(4)$ \\
\hline N1-W1-C7 & $134.88(13)$ \\
\hline
\end{tabular}




\begin{tabular}{|c|c|}
\hline N2-W1-C7 & $31.77(12)$ \\
\hline N3-W1-C7 & $29.20(11)$ \\
\hline $\mathrm{Cl3}-\mathrm{W} 1-\mathrm{C} 7$ & $122.35(9)$ \\
\hline Cl1-W1-C7 & $88.32(8)$ \\
\hline $\mathrm{Cl} 2-\mathrm{W} 1-\mathrm{C} 7$ & $86.15(8)$ \\
\hline N4'-W2-N4 & $16.6(4)$ \\
\hline N4'-W2-N6 & $99.0(5)$ \\
\hline N4-W2-N6 & $106.4(5)$ \\
\hline N4'-W2-N5 & $158.6(5)$ \\
\hline N4-W2-N5 & $166.0(6)$ \\
\hline N6-W2-N5 & $61.04(12)$ \\
\hline N4'-W2-Cl6 & $106.1(5)$ \\
\hline N4-W2-C16 & $98.7(5)$ \\
\hline N6-W2-C16 & $154.67(10)$ \\
\hline N5-W2-C16 & $93.63(9)$ \\
\hline N4'-W2-C15 & $88.3(5)$ \\
\hline N4-W2-C15 & $102.9(4)$ \\
\hline N6-W2-C15 & $90.95(10)$ \\
\hline N5-W2-C15 & $84.40(9)$ \\
\hline Cl6-W2-C15 & $86.84(4)$ \\
\hline N4'-W2-Cl4 & $104.1(4)$ \\
\hline N4-W2-C14 & $89.0(4)$ \\
\hline N6-W2-C14 & $90.75(10)$ \\
\hline
\end{tabular}




\begin{tabular}{|c|c|}
\hline N5-W2-C14 & $85.13(9)$ \\
\hline Cl6-W2-C14 & $86.11(4)$ \\
\hline $\mathrm{Cl} 15-\mathrm{W} 2-\mathrm{Cl} 4$ & $166.98(5)$ \\
\hline N4'-W2-C18 & $130.4(5)$ \\
\hline N4-W2-C18 & $137.9(5)$ \\
\hline N6-W2-C18 & $31.81(12)$ \\
\hline N5-W2-C18 & $29.24(11)$ \\
\hline C16-W2-C18 & $122.87(9)$ \\
\hline C15-W2-C18 & $86.92(9)$ \\
\hline Cl4-W2-C18 & $87.73(8)$ \\
\hline C1-N1-W1 & $174.0(3)$ \\
\hline C7-N2-C4 & $121.2(3)$ \\
\hline C7-N2-W1 & $100.9(2)$ \\
\hline C4-N2-W1 & $137.8(3)$ \\
\hline C7-N3-C9 & $125.4(3)$ \\
\hline C7-N3-W1 & $90.1(2)$ \\
\hline C9-N3-W1 & $144.5(2)$ \\
\hline C12-N4-W2 & $176.2(13)$ \\
\hline $\mathrm{C} 12^{\prime}-\mathrm{N} 4{ }^{\prime}-\mathrm{W} 2$ & $172.2(13)$ \\
\hline C18-N5-C15 & $126.6(3)$ \\
\hline C18-N5-W2 & $90.1(2)$ \\
\hline C15-N5-W2 & $143.2(3)$ \\
\hline C18-N6-C20 & $122.6(3)$ \\
\hline
\end{tabular}




\begin{tabular}{|c|c|}
\hline C18-N6-W2 & $100.9(2)$ \\
\hline C20-N6-W2 & $136.5(3)$ \\
\hline $\mathrm{N} 1-\mathrm{C} 1-\mathrm{C} 3$ & $109.3(4)$ \\
\hline $\mathrm{N} 1-\mathrm{C} 1-\mathrm{C} 2$ & $110.8(4)$ \\
\hline $\mathrm{C} 3-\mathrm{C} 1-\mathrm{C} 2$ & $112.9(4)$ \\
\hline N1-C1-H1A & 107.9 \\
\hline C3-C1-H1A & 107.9 \\
\hline $\mathrm{C} 2-\mathrm{C} 1-\mathrm{H} 1 \mathrm{~A}$ & 107.9 \\
\hline $\mathrm{C} 1-\mathrm{C} 2-\mathrm{H} 2 \mathrm{~A}$ & 109.5 \\
\hline $\mathrm{C} 1-\mathrm{C} 2-\mathrm{H} 2 \mathrm{~B}$ & 109.5 \\
\hline $\mathrm{H} 2 \mathrm{~A}-\mathrm{C} 2-\mathrm{H} 2 \mathrm{~B}$ & 109.5 \\
\hline $\mathrm{C} 1-\mathrm{C} 2-\mathrm{H} 2 \mathrm{C}$ & 109.5 \\
\hline $\mathrm{H} 2 \mathrm{~A}-\mathrm{C} 2-\mathrm{H} 2 \mathrm{C}$ & 109.5 \\
\hline $\mathrm{H} 2 \mathrm{~B}-\mathrm{C} 2-\mathrm{H} 2 \mathrm{C}$ & 109.5 \\
\hline C1-C3-H3A & 109.5 \\
\hline $\mathrm{C} 1-\mathrm{C} 3-\mathrm{H} 3 \mathrm{~B}$ & 109.5 \\
\hline H3A-C3-H3B & 109.5 \\
\hline $\mathrm{C} 1-\mathrm{C} 3-\mathrm{H} 3 \mathrm{C}$ & 109.5 \\
\hline $\mathrm{H} 3 \mathrm{~A}-\mathrm{C} 3-\mathrm{H} 3 \mathrm{C}$ & 109.5 \\
\hline H3B-C3-H3C & 109.5 \\
\hline N2-C4-C6 & $109.9(4)$ \\
\hline N2-C4-C5 & $111.0(3)$ \\
\hline C6-C4-C5 & $112.8(4)$ \\
\hline
\end{tabular}




\begin{tabular}{|c|c|}
\hline N2-C4-H4A & 107.6 \\
\hline C6-C4-H4A & 107.6 \\
\hline C5-C4-H4A & 107.6 \\
\hline C4-C5-H5A & 109.5 \\
\hline C4-C5-H5B & 109.5 \\
\hline $\mathrm{H} 5 \mathrm{~A}-\mathrm{C} 5-\mathrm{H} 5 \mathrm{~B}$ & 109.5 \\
\hline C4-C5-H5C & 109.5 \\
\hline $\mathrm{H} 5 \mathrm{~A}-\mathrm{C} 5-\mathrm{H} 5 \mathrm{C}$ & 109.5 \\
\hline H5B-C5-H5C & 109.5 \\
\hline C4-C6-H6A & 109.5 \\
\hline C4-C6-H6B & 109.5 \\
\hline H6А-C6-H6B & 109.5 \\
\hline C4-C6-H6C & 109.5 \\
\hline H6A-C6-H6C & 109.5 \\
\hline H6B-C6-H6C & 109.5 \\
\hline N3-C7-N2 & $108.0(3)$ \\
\hline N3-C7-C8 & $128.6(4)$ \\
\hline $\mathrm{N} 2-\mathrm{C} 7-\mathrm{C} 8$ & $123.3(3)$ \\
\hline N3-C7-W1 & $60.7(2)$ \\
\hline N2-C7-W1 & $47.36(17)$ \\
\hline C8-C7-W1 & $170.5(3)$ \\
\hline C7-C8-H8A & 109.5 \\
\hline C7-C8-H8B & 109.5 \\
\hline
\end{tabular}




\begin{tabular}{|c|c|}
\hline H8A-C8-H8B & 109.5 \\
\hline C7-C8-H8C & 109.5 \\
\hline H8A-C8-H8C & 109.5 \\
\hline H8B-C8-H8C & 109.5 \\
\hline N3-C9-C11 & $109.5(3)$ \\
\hline N3-C9-C10 & $107.8(3)$ \\
\hline C11-C9-C10 & $112.2(4)$ \\
\hline N3-C9-H9A & 109.1 \\
\hline C11-C9-H9A & 109.1 \\
\hline C10-C9-H9A & 109.1 \\
\hline C9-C10-H10A & 109.5 \\
\hline C9-C10-H10B & 109.5 \\
\hline H10A-C10-H10B & 109.5 \\
\hline C9-C10-H10C & 109.5 \\
\hline H10A-C10-H10C & 109.5 \\
\hline H10B-C10-H10C & 109.5 \\
\hline C9-C11-H11A & 109.5 \\
\hline C9-C11-H11B & 109.5 \\
\hline H11A-C11-H11B & 109.5 \\
\hline C9-C11-H11C & 109.5 \\
\hline H11A-C11-H11C & 109.5 \\
\hline H11B-C11-H11C & 109.5 \\
\hline N4-C12-C14 & $114.7(14)$ \\
\hline
\end{tabular}




\begin{tabular}{ll} 
N4-C12-C13 & $106.4(10)$ \\
C14-C12-C13 & $114.2(12)$ \\
N4-C12-H12A & 107.0 \\
C14-C12-H12A & 107.0 \\
C13-C12-H12A & 107.0 \\
C14'-C12'-C13' & $115.8(15)$ \\
C14'-C12'-N4' & $111.9(12)$ \\
C13'-C12'-N4' & $107.8(18)$ \\
C14'-C12'-H12B & 107.0 \\
C13'-C12'-H12B & 107.0 \\
N4'-C12'-H12B & 107.0 \\
C12-C13-H13A & 109.5 \\
C12-C13-H13B & 109.5 \\
H13A-C13-H13B & 109.5 \\
C12-C13-H13C & 109.5 \\
H13A-C13-H13C & 109.5 \\
H13B-C13-H13C & 109.5 \\
C12'-C13'-H13D & 109.5 \\
C12'-C13'-H13E & 109.5 \\
H13D-C13'-H13E & 109.5 \\
C12'-C13'-H13F & 109.5 \\
H13D-C13'-H13F & 109.5 \\
H13E-C13'-H13F & 109.5 \\
\hline
\end{tabular}




\begin{tabular}{|c|c|}
\hline C12-C14-H14A & 109.5 \\
\hline C12-C14-H14B & 109.5 \\
\hline H14A-C14-H14B & 109.5 \\
\hline C12-C14-H14C & 109.5 \\
\hline H14A-C14-H14C & 109.5 \\
\hline H14B-C14-H14C & 109.5 \\
\hline C12'-C14'-H14D & 109.5 \\
\hline C12'-C14'-H14E & 109.5 \\
\hline H14D-C14'-H14E & 109.5 \\
\hline C12'-C14'-H14F & 109.5 \\
\hline H14D-C14'-H14F & 109.5 \\
\hline H14E-C14'-H14F & 109.5 \\
\hline N5-C15-C17 & $109.5(4)$ \\
\hline N5-C15-C16 & $107.7(4)$ \\
\hline C17-C15-C16 & $112.4(4)$ \\
\hline N5-C15-H15A & 109.0 \\
\hline C17-C15-H15A & 109.0 \\
\hline C16-C15-H15A & 109.0 \\
\hline C15-C16-H16A & 109.5 \\
\hline C15-C16-H16B & 109.5 \\
\hline H16A-C16-H16B & 109.5 \\
\hline C15-C16-H16C & 109.5 \\
\hline H16A-C16-H16C & 109.5 \\
\hline
\end{tabular}




\begin{tabular}{ll} 
H16B-C16-H16C & 109.5 \\
C15-C17-H17A & 109.5 \\
C15-C17-H17B & 109.5 \\
H17A-C17-H17B & 109.5 \\
C15-C17-H17C & 109.5 \\
H17A-C17-H17C & 109.5 \\
H17B-C17-H17C & 109.5 \\
N5-C18-N6 & $107.9(3)$ \\
N5-C18-C19 & $129.7(4)$ \\
N6-C18-C19 & $122.4(3)$ \\
N5-C18-W2 & $60.6(2)$ \\
N6-C18-W2 & $47.29(17)$ \\
C19-C18-W2 & $169.7(3)$ \\
C18-C19-H19A & 109.5 \\
C18-C19-H19B & 109.5 \\
H19A-C19-H19B & 109.5 \\
C18-C19-H19C & 109.5 \\
H19A-C19-H19C & 109.5 \\
H19B-C19-H19C & 109.5 \\
N6-C20-C22 & $109.3(4)$ \\
N6-C20-C21 & $109.8(4)$ \\
N6-C22-C20-C21 & $112.7(4)$ \\
& 108.4 \\
\hline
\end{tabular}




\begin{tabular}{lr} 
C22-C20-H20A & 108.4 \\
C21-C20-H20A & 108.4 \\
C20-C21-H21A & 109.5 \\
C20-C21-H21B & 109.5 \\
H21A-C21-H21B & 109.5 \\
C20-C21-H21C & 109.5 \\
H21A-C21-H21C & 109.5 \\
H21B-C21-H21C & 109.5 \\
C20-C22-H22A & 109.5 \\
C20-C22-H22B & 109.5 \\
H22A-C22-H22B & 109.5 \\
C20-C22-H22C & 109.5 \\
H22A-C22-H22C & 109.5 \\
H22B-C22-H22C & 109.5 \\
\hline
\end{tabular}

Symmetry transformations used to generate equivalent atoms: 
Table S9. Anisotropic displacement parameters $\left(\AA^{2} \times 10^{3}\right)$ for 9 . The anisotropic displacement factor exponent takes the form: $-2 \pi^{2}\left[h^{2} a^{* 2} U^{11}+\ldots+2 h k a^{*} b^{*} U^{12}\right]$

\begin{tabular}{|c|c|c|c|c|c|c|}
\hline & $\mathrm{U}^{11}$ & $\mathrm{U}^{22}$ & U33 & $\mathrm{U}^{23}$ & $\mathrm{U}^{13}$ & $\mathrm{U}^{12}$ \\
\hline W1 & $34(1)$ & $33(1)$ & $27(1)$ & $-2(1)$ & $16(1)$ & $-1(1)$ \\
\hline W2 & $34(1)$ & $46(1)$ & $32(1)$ & $3(1)$ & $17(1)$ & $-6(1)$ \\
\hline Cl1 & $41(1)$ & $34(1)$ & $44(1)$ & $-2(1)$ & $19(1)$ & $-5(1)$ \\
\hline $\mathrm{Cl} 2$ & $54(1)$ & $50(1)$ & $75(1)$ & $2(1)$ & $34(1)$ & $-12(1)$ \\
\hline $\mathrm{Cl} 3$ & $68(1)$ & 71(1) & $31(1)$ & $-4(1)$ & $29(1)$ & $-2(1)$ \\
\hline $\mathrm{Cl} 4$ & $75(1)$ & $48(1)$ & $66(1)$ & 11(1) & $44(1)$ & $11(1)$ \\
\hline $\mathrm{Cl} 5$ & $51(1)$ & $81(1)$ & $53(1)$ & $8(1)$ & $29(1)$ & $20(1)$ \\
\hline $\mathrm{Cl} 6$ & $52(1)$ & $70(1)$ & $35(1)$ & 11(1) & 21(1) & $0(1)$ \\
\hline $\mathrm{N} 1$ & $35(2)$ & $37(2)$ & $38(2)$ & $-4(1)$ & $21(1)$ & $-1(1)$ \\
\hline N2 & $39(2)$ & $35(2)$ & $25(2)$ & $-4(1)$ & $13(1)$ & $3(1)$ \\
\hline N3 & $34(2)$ & $27(1)$ & $29(2)$ & $0(1)$ & $14(1)$ & $-1(1)$ \\
\hline N4 & $34(6)$ & $40(7)$ & $49(5)$ & $27(5)$ & $21(5)$ & $6(5)$ \\
\hline N4' & $31(6)$ & $30(7)$ & $33(5)$ & $10(5)$ & $11(5)$ & $-1(5)$ \\
\hline N5 & $36(2)$ & $39(2)$ & $37(2)$ & $1(2)$ & $15(2)$ & $-3(1)$ \\
\hline N6 & $38(2)$ & $44(2)$ & $30(2)$ & $-1(1)$ & $16(1)$ & $-7(1)$ \\
\hline $\mathrm{C} 1$ & $34(2)$ & $42(2)$ & $52(2)$ & $2(2)$ & $19(2)$ & $6(2)$ \\
\hline $\mathrm{C} 2$ & $35(3)$ & $76(4)$ & $140(6)$ & $-14(4)$ & $17(3)$ & $-6(3)$ \\
\hline $\mathrm{C} 3$ & $72(4)$ & $84(4)$ & $86(4)$ & $-30(3)$ & $23(3)$ & $28(3)$ \\
\hline $\mathrm{C} 4$ & $49(3)$ & $49(2)$ & $27(2)$ & $-4(2)$ & $10(2)$ & $9(2)$ \\
\hline
\end{tabular}




\begin{tabular}{|c|c|c|c|c|c|c|}
\hline $\mathrm{C} 5$ & $69(3)$ & $59(3)$ & $27(2)$ & $6(2)$ & $12(2)$ & $2(2)$ \\
\hline C6 & $80(4)$ & $68(3)$ & $46(3)$ & $-3(3)$ & $-11(3)$ & $-15(3)$ \\
\hline $\mathrm{C} 7$ & $36(2)$ & $31(2)$ & $35(2)$ & $-4(2)$ & $17(2)$ & $-3(2)$ \\
\hline $\mathrm{C} 8$ & $54(3)$ & $39(2)$ & $36(2)$ & $-7(2)$ & $17(2)$ & $8(2)$ \\
\hline C9 & $41(2)$ & $36(2)$ & $38(2)$ & $3(2)$ & $16(2)$ & $5(2)$ \\
\hline $\mathrm{C} 10$ & $60(3)$ & $49(2)$ & $50(3)$ & $17(2)$ & $22(2)$ & $4(2)$ \\
\hline $\mathrm{C} 11$ & $44(3)$ & $54(3)$ & $41(2)$ & $3(2)$ & $8(2)$ & $-1(2)$ \\
\hline $\mathrm{C} 12$ & $28(5)$ & $57(8)$ & $60(6)$ & $12(6)$ & $11(4)$ & $-13(5)$ \\
\hline $\mathrm{C} 12^{\prime}$ & 49(9) & $61(8)$ & $68(8)$ & $-1(7)$ & $28(7)$ & $-12(7)$ \\
\hline C13 & $112(10)$ & $61(7)$ & $80(8)$ & $11(6)$ & $59(7)$ & $-28(7)$ \\
\hline C13' & $230(30)$ & $83(13)$ & $490(70)$ & $-120(30)$ & $290(40)$ & $-95(19)$ \\
\hline C14 & $30(7)$ & $117(14)$ & $190(20)$ & $71(14)$ & $-19(9)$ & $-16(8)$ \\
\hline C14' & $67(9)$ & $137(13)$ & $139(16)$ & $-53(12)$ & $63(10)$ & $-33(9)$ \\
\hline $\mathrm{C} 15$ & $48(3)$ & $56(3)$ & $43(2)$ & $8(2)$ & $13(2)$ & $-16(2)$ \\
\hline $\mathrm{C} 16$ & $77(4)$ & $48(3)$ & $53(3)$ & $-9(2)$ & $-1(3)$ & $2(3)$ \\
\hline $\mathrm{C} 17$ & $39(3)$ & $84(3)$ & $53(3)$ & $7(3)$ & $14(2)$ & $-1(2)$ \\
\hline $\mathrm{C} 18$ & $27(2)$ & $38(2)$ & $37(2)$ & $4(2)$ & $16(2)$ & $3(2)$ \\
\hline C19 & $47(2)$ & $47(2)$ & $45(2)$ & $17(2)$ & $22(2)$ & $2(2)$ \\
\hline $\mathrm{C} 20$ & $51(3)$ & $63(3)$ & $31(2)$ & $3(2)$ & $19(2)$ & $-8(2)$ \\
\hline $\mathrm{C} 21$ & $69(3)$ & $72(3)$ & $46(3)$ & $-18(2)$ & $26(2)$ & $-20(3)$ \\
\hline $\mathrm{C} 22$ & $66(3)$ & $89(4)$ & $47(3)$ & $10(3)$ & $2(3)$ & $14(3)$ \\
\hline
\end{tabular}


Table S10. Hydrogen coordinates $\left(x 10^{4}\right)$ and isotropic displacement parameters $\left(\AA^{2} \times 10^{3}\right)$ for 9.

\begin{tabular}{|c|c|c|c|c|}
\hline & $\mathrm{x}$ & $\mathrm{y}$ & z & $\mathrm{U}(\mathrm{eq})$ \\
\hline $\mathrm{H} 1 \mathrm{~A}$ & 2940 & 9877 & 2543 & 51 \\
\hline $\mathrm{H} 2 \mathrm{~A}$ & 2048 & 8513 & 2188 & 135 \\
\hline $\mathrm{H} 2 \mathrm{~B}$ & 1579 & 9364 & 2476 & 135 \\
\hline $\mathrm{H} 2 \mathrm{C}$ & 2044 & 8504 & 3129 & 135 \\
\hline $\mathrm{H} 3 \mathrm{~A}$ & 3576 & 10457 & 3952 & 126 \\
\hline H3B & 3003 & 9721 & 4234 & 126 \\
\hline $\mathrm{H} 3 \mathrm{C}$ & 2528 & 10569 & 3571 & 126 \\
\hline $\mathrm{H} 4 \mathrm{~A}$ & 4488 & 7276 & 1442 & 52 \\
\hline $\mathrm{H} 5 \mathrm{~A}$ & 4433 & 8942 & 1623 & 82 \\
\hline H5B & 3499 & 8835 & 1678 & 82 \\
\hline $\mathrm{H} 5 \mathrm{C}$ & 3590 & 8591 & 798 & 82 \\
\hline H6A & 3472 & 6247 & 1637 & 115 \\
\hline H6B & 2994 & 6910 & 806 & 115 \\
\hline $\mathrm{H} 6 \mathrm{C}$ & 2898 & 7150 & 1685 & 115 \\
\hline H8A & 6141 & 5838 & 2894 & 65 \\
\hline H8B & 5688 & 6515 & 2068 & 65 \\
\hline $\mathrm{H} 8 \mathrm{C}$ & 5138 & 5660 & 2242 & 65 \\
\hline
\end{tabular}




\begin{tabular}{|c|c|c|c|}
\hline H9A & 6592 & 5929 & 4166 \\
\hline H10A & 5502 & 5259 & 4559 \\
\hline H10B & 5675 & 6078 & 5271 \\
\hline $\mathrm{H} 10 \mathrm{C}$ & 6421 & 5297 & 5387 \\
\hline $\mathrm{H} 11 \mathrm{~A}$ & 7238 & 7413 & 4736 \\
\hline H11B & 7498 & 6639 & 5496 \\
\hline $\mathrm{H} 11 \mathrm{C}$ & 6751 & 7420 & 5376 \\
\hline $\mathrm{H} 12 \mathrm{~A}$ & 10622 & 9646 & 9630 \\
\hline H12B & 11475 & 8494 & 10055 \\
\hline $\mathrm{H} 13 \mathrm{~A}$ & 10089 & 9820 & 8152 \\
\hline H13B & 10840 & 9119 & 8122 \\
\hline $\mathrm{H} 13 \mathrm{C}$ & 11102 & 10138 & 8595 \\
\hline H13D & 10597 & 9798 & 9697 \\
\hline $\mathrm{H} 13 \mathrm{E}$ & 10595 & 9806 & 8754 \\
\hline $\mathrm{H} 13 \mathrm{~F}$ & 11491 & 10001 & 9583 \\
\hline $\mathrm{H} 14 \mathrm{~A}$ & 11670 & 8525 & 10371 \\
\hline H14B & 12078 & 9348 & 9987 \\
\hline $\mathrm{H} 14 \mathrm{C}$ & 11848 & 8319 & 9529 \\
\hline H14D & 11730 & 7613 & 9091 \\
\hline H14E & 12184 & 8647 & 9195 \\
\hline $\mathrm{H} 14 \mathrm{~F}$ & 11294 & 8419 & 8369 \\
\hline H15A & 7206 & 5492 & 8286 \\
\hline H16A & 8197 & 4883 & 7730 \\
\hline
\end{tabular}




\begin{tabular}{|c|c|c|c|c|}
\hline H16B & 7997 & 5776 & 7084 & 102 \\
\hline $\mathrm{H} 16 \mathrm{C}$ & 7243 & 5002 & 6959 & 102 \\
\hline H17A & 6571 & 7011 & 7883 & 90 \\
\hline H17B & 6241 & 6328 & 7053 & 90 \\
\hline $\mathrm{H} 17 \mathrm{C}$ & 6990 & 7112 & 7190 & 90 \\
\hline H19A & 7727 & 5356 & 9601 & 69 \\
\hline H19B & 8183 & 6064 & 10405 & 69 \\
\hline H19C & 8736 & 5201 & 10247 & 69 \\
\hline $\mathrm{H} 20 \mathrm{~A}$ & 9541 & 6624 & 11022 & 57 \\
\hline $\mathrm{H} 21 \mathrm{~A}$ & 9327 & 8296 & 10870 & 92 \\
\hline $\mathrm{H} 21 \mathrm{~B}$ & 10205 & 8412 & 10708 & 92 \\
\hline $\mathrm{H} 21 \mathrm{C}$ & 10267 & 8070 & 11630 & 92 \\
\hline $\mathrm{H} 22 \mathrm{~A}$ & 10645 & 5867 & 10719 & 111 \\
\hline H22B & 11087 & 6559 & 11536 & 111 \\
\hline $\mathrm{H} 22 \mathrm{C}$ & 11031 & 6889 & 10613 & 111 \\
\hline
\end{tabular}

